\title{
POG Neuroblastoma Stage B
}

National Cancer Institute

\section{Source}

National Cancer Institute. POG Neuroblastoma Stage B. NCI Thesaurus. Code C85425.

The primary tumor is grossly incompletely resected; lymph nodes and liver are not involved by tumor. 